\title{
Evaluation of Duration of Immunogenicity and Protective Efficacy of Improved Influenza Viral Vector-Based Brucella abortus Vaccine Against Brucella melitensis Infection in Sheep and Goats
}

\section{OPEN ACCESS \\ Edited by: \\ Maxim C.-J. Cheeran,}

University of Minnesota, United States

Reviewed by:

Angel Alejandro Oñate, University of Concepcion, Chile

Steven Olsen,

United States Department of Agriculture (USDA), United States

Sean Ranjan Wattegedera,

Moredun Research Institute, United Kingdom

${ }^{*}$ Correspondence: Kaissar Tabynov

ktabynov@gmail.com; tabynov_81@mail.ru

Specialty section:

This article was submitted to Veterinary Infectious Diseases,

a section of the journa

Frontiers in Veterinary Science

Received: 28 October 2019

Accepted: 23 January 2020

Published: 27 February 2020

Citation:

Mailybayeva A, Ryskeldinova S,

Zinina N, Zhou E-M, Renukaradhya GJ and Tabynov K (2020) Evaluation of Duration of Immunogenicity and

Protective Efficacy of Improved

Influenza Viral Vector-Based Brucella abortus Vaccine Against Brucella melitensis Infection in Sheep and

Goats. Front. Vet. Sci. 7:58

doi: 10.3389/fvets.2020.00058

\author{
Aigerim Mailybayeva ${ }^{1}$, Sholpan Ryskeldinova ${ }^{1}$, Nadezhda Zinina ${ }^{2}$, En-Min Zhou ${ }^{3}$, \\ Gourapura J. Renukaradhya ${ }^{4}$ and Kaissar Tabynov ${ }^{5,6 *}$
}

1 Laboratory of Infectious Disease Prevention, Research Institute for Biological Safety Problems, Gvardeiskiy, Kazakhstan, ${ }^{2}$ Laboratory of Microbiology, Research Institute for Biological Safety Problems, Gvardeiskiy, Kazakhstan, ${ }^{3}$ Department of Preventive Veterinary Medicine, College of Veterinary Medicine, Northwest A\&F University, Yangling, China, ${ }^{4}$ Food Animal Health Research Program, Department of Veterinary Preventive Medicine, College of Food Agricultural and Environmental Sciences, The Ohio State University, Wooster, OH, United States, ${ }^{5}$ Biological Safety Department, Kazakh National Agrarian University, Almaty, Kazakhstan, ${ }^{6}$ General Clinical Department, Research Institute of Cardiology and Internal Medicine, Almaty, Kazakhstan

In this study, we first evaluated the duration of a protective immune response against Brucella melitensis infection in non-pregnant sheep and goats immunized with an improved (by vaccine formulation and route of administration) commercial Brucella abortus vaccine based on influenza viral vectors expressing Brucella immunodominant Omp16, L7/L12, Omp19, or Cu-Zn superoxide dismutase (SOD) proteins (Flu-BA_Omp19-SOD). Sheep and goats in the vaccinated group were immunized thrice concurrently via the subcutaneous and conjunctival routes of administration at an interval of 21 days. Animals in the control group were administered with 20\% Montanide Gel01 adjuvant in phosphate-buffered saline in the same way. We showed that the Flu-BA_Omp19-SOD vaccine in sheep and goats induces antigen-specific Th1-biased [immunoglobulin G2a (lgG2a) over lgG1] antibody response and T-cell and interferon $\gamma$ responses lasting over a period of 1 month post-last vaccination (PLV). The levels of protection against $B$. melitensis $16 \mathrm{M}$ infection (vaccination efficacy) in vaccinated sheep for a period of 6 months were $0-20 \%$ and in goats $20-40 \%$ compared to control challenge group. But the severity of $B$. melitensis $16 \mathrm{M}$ infection in the Flu-BA_Omp19-SOD-vaccinated sheep and goats during the entire period of observation revealed the infection index $(P=0.001-P<0.0001)$ and Brucella colonization in lymph nodes and organs $(P=0.04-P<0.0001)$ were significantly lower than those in the control group. To conclude, the Flu-BA_Omp19-SOD vaccine using improved formulation and administration method in sheep and goats provides augmented antigen specific humoral and T-cell immune response lasting only for 1 month PLV and partial protection for 6 months against B. melitensis $16 \mathrm{M}$ infection.

Keywords: Brucella melitensis, influenza viral vector, vaccine, T-cell-mediated immune response, prolonged efficacy, small ruminants 


\section{INTRODUCTION}

Brucellosis is a chronic infectious disease of animals and humans that induces huge economic losses globally. Brucella melitensis is the causative agent of brucellosis in sheep and goats and represents the greatest risk to human health among all known Brucella species (1). To control brucellosis in animals, vaccination is one of the most cost-effective measures, which in turn helps in protecting the health of humans in endemic areas (2). This also aids in eradication of the disease among livestock (3). Currently, attenuated B. melitensis Rev.1 vaccine is used in sheep and goats (4). Although the Rev.1 vaccine has been found effective, it has several limitations such as it causes abortion in a fraction of vaccinated animals, the vaccine bacteria are virulent to humans, and differentiation of infected from vaccinated animals (DIVA) is a challenge $(4,5)$. Therefore, development of a safe and effective vaccine to control B. melitensis infection in sheep and goats that has DIVA potential is warranted.

Earlier, we developed a novel Brucella abortus vaccine based on influenza viral vector (IVV) expressing Brucellaimmunodominant outer membrane protein (Omp)16 or ribosomal L7/L12 protein (Flu-BA) (6). In January 2019, the Flu-BA vaccine was registered in Kazakhstan (registration certificate no. RK-VP-1-3775-19 dated January 14, 2019) and is currently being used in cattle against $B$. abortus infection. The vaccine response data obtained in cattle (6), as well as information supporting the ability of influenza viruses to infect sheep and goats $(7,8)$, suggest that vaccines based on IVV can be an effective candidate in small ruminants. It is important to note that the IVV-expressing proteins are immunodominant and common (genetically similar for 95-99\%) for B. melitensis, B. abortus, Brucella suis, and Brucella canis (9-11). Our earlier study with Flu-BA vaccine provided 57.1 and $42.9 \%$ efficacy in vaccinated non-pregnant sheep and goats, respectively (12), which prompted us to evaluate the improved Flu-BA vaccine formulation. This formulation had additional IVV-expressing Brucella Omp19 and $\mathrm{Cu}, \mathrm{Zn}$ superoxide dismutase (SOD) proteins, an increased concentration of the adjuvant Montanide Gel01 by 2-fold called Flu-BA_Omp19-SOD, and delivery system (administered the vaccine simultaneously by subcutaneous and conjunctival routes), and the number of doses was increased to three from two and tested in pregnant sheep and goats against $B$. melitensis challenge infection. In pregnant small ruminants, the Flu-BA_Omp19-SOD vaccine was shown to be safe and effective with complete protection (lack of Brucella isolation in all animal samples) against B. melitensis infection in $66.7 \%$ sheep and $55.6 \%$ goats (12), whereas the commercial Rev.1 vaccine provides protection against B. melitensis infection in $83.3 \%$ goats and $100 \%$ sheep (12). Because of added benefits of the Flu-BA_Omp19-SOD vaccine, it is considered as a promising candidate. However, it was important to define the extended duration of protective efficacy of the Flu-BA_Omp19-SOD in sheep and goats, which was the objective of this study. The ability of a vaccine to form a long-term protective immune response is one of its most valuable and critical properties, and therefore this research has been decisive in continuing or discontinuing work in this area.

\section{MATERIALS AND METHODS}

\section{Bacterial Strains and Biosafety Aspects}

The virulent strain B. melitensis $16 \mathrm{M}$ (obtained from the Research Institute for Biological Safety Problem's collection of microorganisms) was used in this study. The bacterial cells were cultured under aerobic conditions in Brucella base agar (Sigma, St. Louis, MO, USA) at $37^{\circ} \mathrm{C}$. All experiments with live Brucella were performed in level 3 biosafety facilities. Challenged sheep and goats were contained in specialized facilities (biosafety level 3 agricultural).

\section{Vaccine Preparation}

Vaccines were prepared from the four IVV subtypes A/H5N1 expressing the Brucella L7/L12 or Omp16 or Omp19 or Cu-Zn SOD (SOD) proteins from the open reading frame of the NS1 gene (Flu-NS1-124-L7/L12-H5N1, Flu-NS1-124-Omp16-H5N1, Flu-NS1-124-Omp19-H5N1, Flu-NS1-124-SOD-H5N1), which were generated previously (13). A detailed description of the vaccine preparation procedure is described previously (14). An improved tetravalent vaccine formulation expressing the Brucella L7/L12, Omp16, Omp19, SOD proteins was provisionally referred to as the Flu-BA_Omp19-SOD. The lyophilized vaccine before administration was resuspended $(2.5 \mathrm{~mL}$ per ampule) in a $20 \%$ solution of the adjuvant Montanide Gel01 (Seppic, Puteaux, France) in phosphate-buffered saline (PBS).

\section{Vaccination, Study Design, and Sampling}

A total of 30 Degeresskaya semifine meat and wool purpose breed of sheep and 30 Gorno-Altaisk breed of goats aged 6-18 months from an officially brucellosis-free flocks were used in this study. All animals were non-pregnant females. Further, two groups were formed from each animal species by randomization method: the Flu-BA_Omp19-SOD-vaccinated and control groups ( $n=15$ /group). Sheep and goats in the vaccinated group were immunized thrice concurrently via the subcutaneous $(2.0 \mathrm{~mL}$ in the axillary region) and conjunctival $(0.25 \mathrm{~mL}$ to each eye) routes of administration at an interval of 21 days with the vaccine dose $7.0 \log _{10} \mathrm{EID}_{50} /$ animal. Animals in the control group were administered with 20\% Montanide Gel01 adjuvant in PBS in the same way. The antigen-specific enzyme-linked immunosorbent assay (ELISA) antibodies (total IgG, IgG2a, and IgG1), lymphocytes stimulation index (SI), and interferon $\gamma$ (IFN- $\gamma$ ) production in animals both before and at the first ( $n=15 /$ group), third ( $n=10 /$ group), and sixth ( $n=5$ /group) months post-last (third dose) vaccination (PLV) in serum and whole-blood samples were performed. At the first ( $n=5$ /group), third ( $n=5$ /group), and sixth ( $n=5$ /group) months PLV, sheep and goats from the vaccinated and control groups were challenged with a virulent strain of B. melitensis $16 \mathrm{M}$ at a dose of $10^{6}$ colony-forming units (CFU)/animal subcutaneously (axillary region right side). At 28 days after challenge, the animals were euthanized and slaughtered aseptically for sampling. Schematic representation of the study design is shown in Figure 1. 


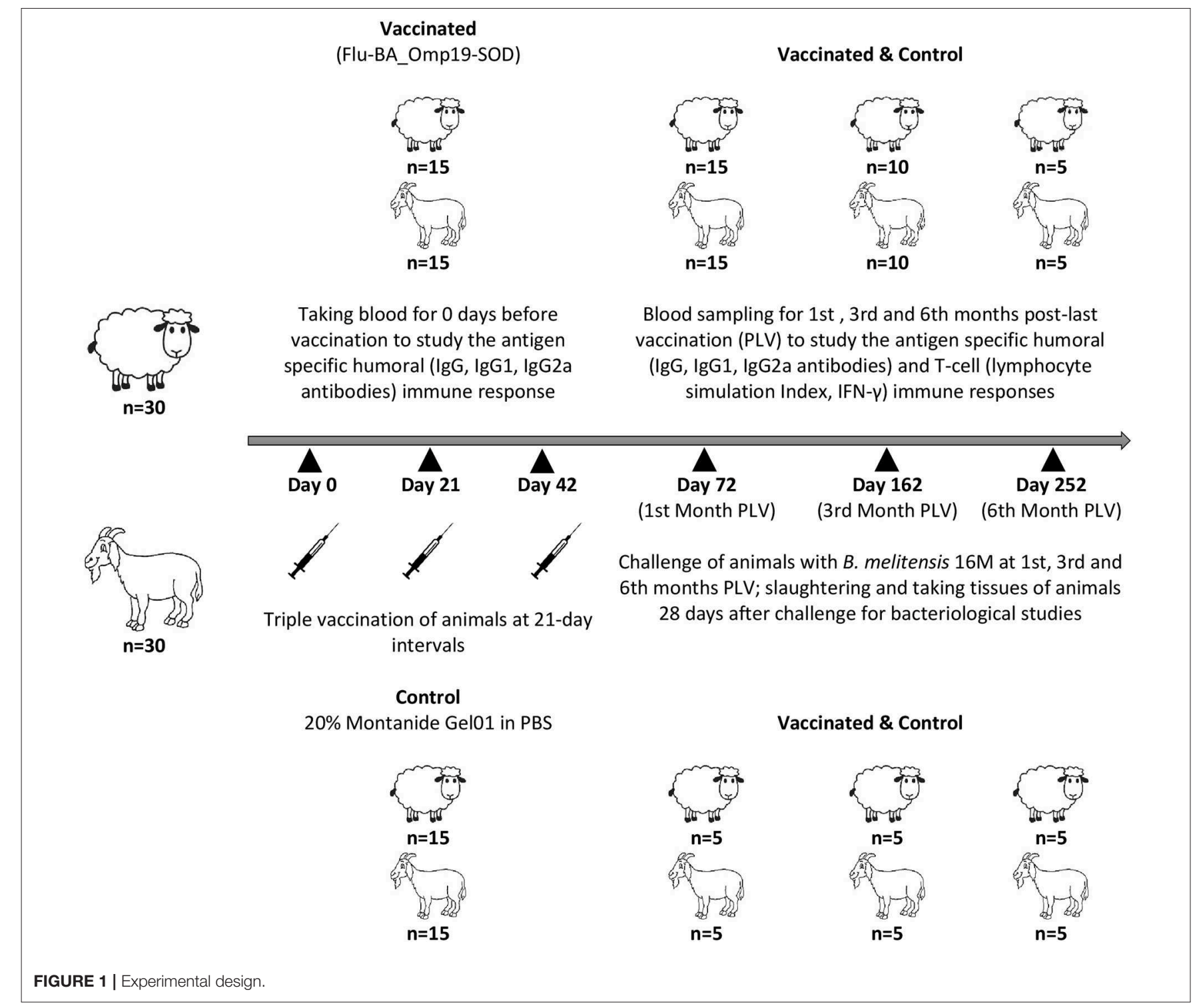

\section{Antibody Response}

This study was conducted in full accordance with our previously published work (14). Briefly, 96-well microtiter plates (Nunc, Roskilde, Denmark) were coated overnight with pre-titrated mixture of Brucella L7/L12, Omp16, Omp19, or SOD proteins (each at $2 \mu \mathrm{g} / \mathrm{mL}$ ) in PBS, blocked for $1 \mathrm{~h}$ using PBS containing $1 \%$ ovalbumin (PBS-OVA; $200 \mu \mathrm{L} /$ well), and washed with PBS containing $0.05 \%$ Tween-20 (PBS/Tw). Serial 2-fold dilutions of the serum samples in PBS/OVA were added $(100 \mu \mathrm{L} /$ well $)$ to the plates and incubated for $1 \mathrm{~h}$ at room temperature. Donkey antiruminant IgG horseradish peroxidase conjugate (Sigma) and monoclonal antibodies specific for sheep IgG1 and IgG2 (Novus Biologicals, Littleton, CO, USA) were used for detection of total and isotype-specific antibodies. After 90 -min incubation at $37^{\circ} \mathrm{C}$, plates were washed, and the specific reactivity was determined by addition of an enzyme substrate ABTS [2,2_azinobis(3ethylbenzthiazolinesulfonic acid)] diammonium (Moss, Inc.,
Pasadena, CA, USA) at $100 \mathrm{~mL} /$ well. The absorbance values were measured at $415 \mathrm{~nm}$. Antibody levels were expressed as the arithmetic mean \pm standard errors of the optical density (OD) value obtained for sheep and goats samples included in each group.

\section{Preparation of Peripheral Blood Mononuclear Cell for Lymphocyte Proliferation Assay}

This work was carried out in accordance with Mailybayeva et al. (14). Briefly, peripheral blood mononuclear cells (PBMCs) were isolated by density gradient centrifugation using the Ficoll-sodium diatrizoate gradient (DNA-Technology, Moscow, Russia). Cells number was adjusted to $10^{7}$ viable cells per $\mathrm{mL}$ determined by trypan blue dye exclusion, and $50 \mu \mathrm{L}$ of each cell suspension (containing $5 \times 10^{5}$ cells) was added to eight 
separate flat-bottomed wells of 96-well microtiter plates already plated with $100 \mu \mathrm{L}$ of RPMI-1640 medium only or RPMI1640 medium containing $8.0 \mu \mathrm{g}$ of purified Brucella proteins L7/L12, Omp16, Omp19, or SOD per well. The cell cultures were incubated for 7 days at $37^{\circ} \mathrm{C}$ under $5 \% \mathrm{CO}_{2}$. After incubation, the cells were pulsed with $1.0 \mu \mathrm{Ci}$ of $\left[{ }^{3} \mathrm{H}\right]$ thymidine per well for $18 \mathrm{~h}$. Cells were harvested onto glass filter mats and counted for radioactivity in a liquid scintillation counter. Cell proliferation results were converted to SI [counts per minute (cpm) of wells containing antigens/cpm in the absence of antigens] for comparison.

\section{Cytokine IFN- $\gamma$ Production}

This study was also conducted in accordance with Mailybayeva et al. (14). Briefly, PBMCs from each animal were adjusted to $10^{7}$ viable cells per $\mathrm{mL}$ as described in the previous paragraph. Aliquots $(50 \mu \mathrm{L})$ of each cell suspension containing $5 \times$ $10^{5}$ cells were plated and stimulated with Brucella proteins L7/L12, Omp16, Omp19, or SOD as described above. Cell cultures were incubated at $37^{\circ} \mathrm{C}$ under $5 \% \quad \mathrm{CO}_{2}$, and the supernatants were harvested $72 \mathrm{~h}$ later and assayed for IFN- $\gamma$ using a commercial ELISA kit (RayBio1Bovine IFN- $\gamma$ ELISA Kit; RayBiotech, Inc., Norcross, GA, USA). This kit has been shown to cross-react with IFN- $\gamma$ of sheep and goats (15). Antigenspecific IFN- $\gamma$ production was determined for each individual animal by subtracting the background concentration of IFN- $\gamma$ in wells without antigen from the IFN- $\gamma$ concentration in wells with antigen.

\section{Assessment Protective Efficacy of the Vaccine in Sheep and Goats}

Samples of lymph nodes (submandibular, retropharyngeal, right subscapular, left subscapular, mediastinal, bronchial, portal, paraaortic, pelvic, mesenteric, udder) and parenchymal organs (liver, kidney, spleen, and bone marrow) were taken from slaughtered animals. In total, 15 organs were sampled from each animal. Bacteriological studies and evaluation of results were carried out as described in the previous study (12). Briefly, the tissue homogenates in $0.1 \%$ Triton-PBS after 10 -fold serial dilutions were plated onto Brucella base agar plates and incubated at $37^{\circ} \mathrm{C}$ for 2 weeks, and the growth of bacterial colonies was counted periodically during this time. The concentrations of bacteria [colony-forming units (CFU)/g of tissue] in the tissue samples were determined by performing standard plate counts. An animal was considered infected if a Brucella colony was detected from the culture of one or more organs. The results of the bacteriological study were evaluated by the following three parameters: (A) vaccination efficacy or number of animals (expressed in \%) with complete protection against infection (lack of Brucella isolation in all animal samples); (B) generalization of the infectious process or infection index (number of organs and lymph nodes of animals in which Brucella are isolated; the arithmetic mean was given); (C) intensity/severity of the infectious process or the degree of Brucella colonization from samples of lymph nodes and organs (expressed as $\log _{10} \mathrm{CFU} / \mathrm{g}$ of tissue).

\section{Statistical Analysis}

Differences in protective efficacy (complete protection vs. infection in animals) between groups were compared by onesided Fisher exact test at a significance level of $\alpha<0.05$. The significance in antibody responses (IgG, IgG1, and IgG2a), SI, concentration of IFN- $\gamma$, the index of infection, and colonization of Brucella in tissues between groups was analyzed using two-way analysis of variance followed by Sidak's multiple-comparisons test. $P<0.05$ was considered significant. Means are reported with standard errors (SEM). Statistical analysis of all experimental data was performed with GraphPad Prism Software version 6.0 (GraphPad Software Inc., La Jolla, CA, USA).

\section{RESULTS}

\section{Antibody Response to Brucella Proteins in Animals}

In the serum of vaccinated sheep and goats, IgG antibody response to a mixture of Brucella L7/L12, Omp16, Omp19, and SOD proteins was at peak levels after 1 month PLV. In sheep, the antibody levels were significantly higher $(P=0.0007)$ compared to control (Figure 2A). Immunoglobulin $G$ antibody responses in goats did not differ at any of the sampling times. Analysis of isotype specific antibodies in sheep and goats at the first month PLV revealed significantly $(P=0.014-0.02)$ higher IgG2a over IgG1 (Figure 2A), indicating the Th1-polarized immune response. At the third and sixth months PLV in vaccinated sheep and goats, reduced production of antibodies was observed, and the data were not statistically significant $(P=0.33-0.97)$ compared to control animals values.

\section{Lymphocyte Proliferation Responses and IFN- $\gamma$ Production After Vaccination}

At the first month PLV, analysis of antigen (Brucella L7/L12, Omp16, Omp19, and SOD proteins)-specific lymphocyte SI values in PBMC (sheep $2.33 \pm 0.25$; goats $2.73 \pm 0.20$; $P=0.047-0.0009$; Figure 2B) and production of IFN- $\gamma$ (sheep $15.33 \pm 1.36 \mathrm{ng} / \mathrm{mL}$; goats $16.86 \pm 1.20 \mathrm{ng} / \mathrm{mL} ; P<0.0001$; Figure 2C) were significantly higher compared to control animals, indicating the robust $\mathrm{T}$-cell response to vaccine. While at the third and sixth months PLV, although the lymphocyte SI (sheep by $12.5-36.3 \%$, goats by $25.0-27.2 \%$ higher) and IFN- $\gamma$ production (sheep by $27.2-35.1 \%$, goats by $27.4-28.2 \%$ higher) values were higher than those in the control animals, the overall T-cell response in vaccinated sheep and goats was not statistically significant (SI, sheep $1.6 \pm 0.24-2.2 \pm 0.35$, goats $2.0 \pm 0.33-$ $2.2 \pm 0.37, P=0.09-0.97$; IFN- $\gamma$, sheep $12.8 \pm 0.97-13.2 \pm$ $1.07 \mathrm{ng} / \mathrm{mL}$; goats $12.4 \pm 1.56-13.1 \pm 1.4 \mathrm{ng} / \mathrm{mL} ; P=0.052-0.5)$.

\section{Vaccine Protection in Sheep and Goats Against $B$. melitensis Infection}

The duration of the Flu-BA_Omp19-SOD vaccine protective efficacy in sheep and goats against B. melitensis $16 \mathrm{M}$ infection was assessed using parameters such as vaccination efficacy (level of full protection against infection expressed in \%), infection index, and Brucella colonization in tissues and organs. Our 


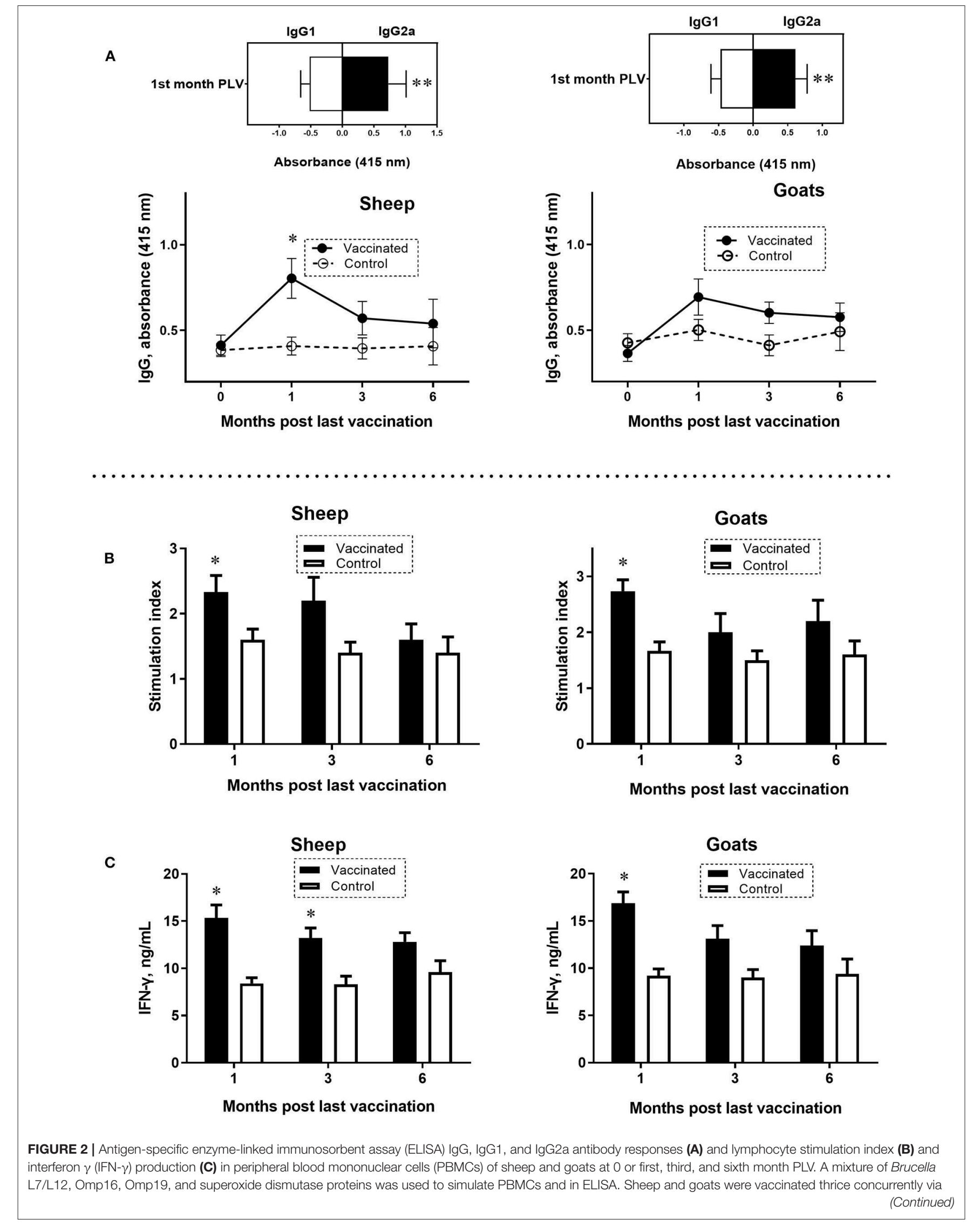


FIGURE 2 | the subcutaneous and conjunctival route. Animals in the control group received only the adjuvant in phosphate-buffered saline. Statistical analysis was performed using two-way analysis of variance followed by Sidak's multiple-comparisons test. ELISA antibody levels were presented as OD \pm standard error. Cell proliferation results were converted to stimulation index [counts per minute (cpm) of wells containing antigens/cpm in the absence of antigens] for comparison. Antigen-specific IFN- $\gamma$ production was determined for each individual animal by subtracting the background concentration of IFN- $\gamma$ in wells without antigen from the IFN- $\gamma$ concentration in wells with antigen. ${ }^{*} P=0.0007$ vaccine group vs. control; ${ }^{\star *} P=0.014-0.02$ IgG2a vs. IgG1 at the first month PLV. Data of lymphocyte stimulation index and levels of IFN- $\gamma$ are presented as mean \pm standard error; ${ }^{*} P=0.047-P<0.0001$ vaccine vs. control group.

TABLE 1 | Rates of infection in the sheep and goats after challenge with the virulent strain Brucella melitensis 16M.

\begin{tabular}{llcccc}
\hline $\begin{array}{l}\text { Post-last } \\
\text { vaccination } \\
\text { period }\end{array}$ & $\begin{array}{c}\text { Group } \\
\text { 1st month }\end{array}$ & $\begin{array}{c}\text { Isolation of } \\
\text { B. melitensis in } \\
\text { sheep, } \boldsymbol{n} \text { (\%) }\end{array}$ & $\begin{array}{c}\text { Total } \\
\text { 3rd month }\end{array}$ & $\begin{array}{c}\text { Isolation of } \\
\text { B. melitensis in } \\
\text { goats, } \boldsymbol{n} \text { (\%) }\end{array}$ & Total \\
& Control & $5(100)$ & 5 & $5(100)$ & 5 \\
& Vaccinated & $5(100)$ & 5 & $4(80)$ & 5 \\
6th month & Control & $5(100)$ & 5 & $5(100)$ & 5 \\
& Vaccinated & $4(80)$ & 5 & $4(80)$ & 5 \\
& Control & $5(100)$ & 5 & $5(100)$ & 5 \\
\hline
\end{tabular}

results showed (Table 1 ) that the levels of protection against B. melitensis $16 \mathrm{M}$ infection (vaccination efficacy) in vaccinated sheep were $0-20 \%$ and $20-40 \%$ in goats compared to the control challenge group (where the animal infection rate was $100 \%$ ), and the data were not statistically significant $(\alpha=0.22-0.5)$. But the severity of $B$. melitensis $16 \mathrm{M}$ infection in the FluBA_Omp19-SOD-vaccinated sheep and goats during the entire period (for up to 6 months PLV) of observation revealed the infection index (Figure 3A: $3.2 \pm 0.9-6.0 \pm 0.8 ; P=0.001-$ $P<0.0001)$ and Brucella colonization in lymph nodes and organs (Figure 3B: $0.1 \pm 0.1-2.0 \pm 0.5 \log _{10} \mathrm{CFU} / \mathrm{g}$ of tissue; $P=0.04-P<0.0001$ ) were significantly lower than those in the control group (infection index 9.8 $\pm 0.6-11.8 \pm 0.5$; Brucella colonization $0.1 \pm 0.1-3.3 \pm 0.2 \log _{10} \mathrm{CFU} / \mathrm{g}$ of tissue). The maximum protection in vaccinated sheep and goats against $B$. melitensis $16 \mathrm{M}$ infection was in the first month PLV. At 1 month PLV compared to the third and sixth months PLV, we observed better vaccination efficacy (20-40\% vs. $0-20 \%)$, a lower index of infection (or number of Brucella isolated organs and lymph nodes in each animal: 3.2-3.4 vs. 3.8-6.0), and degree of Brucella colonization in tissues [the number of lymph nodes and organs where Brucella colonization values were significantly less $(P<0.05)$ than those of the control group: $10-11$ vs. $5-8$ ], indicating that the protective efficacy was reduced after 3 and 6 months PLV.

\section{DISCUSSION}

The Flu-BA vaccine was commercialized to use in cattle in Kazakhstan. Earlier, we tested this vaccine's efficacy after improving the formulation (Flu-BA_Omp19-SOD) and delivery system in pregnant sheep and goats and observed promising safety and efficacy (14). In this study, we for the first time evaluated the duration of protective responses in non-pregnant sheep and goats induced by the candidate vaccine. Earlier, we conducted short-term pilot studies in both non-pregnant and pregnant small ruminants. In this study, the protracted duration of effectiveness of the vaccine for up to 6 months PLV was evaluated.

The protective efficacy of the Flu-BA_Omp19-SOD vaccine in non-pregnant small ruminants at the first month PLV against B. melitensis infection was $20 \%$ in sheep and $40 \%$ in goats, whereas in pregnant sheep and goats, it was 66.7 and $55.6 \%$, respectively (14), wherein similar vaccine formulation and immunization regimen were followed. The severity of B. melitensis infection in vaccinated sheep and goats in this study was measured by infection index (3-3.4 times lower than control) and Brucella colonization in tissues (lower by 131 times than control), which was inferior to those in pregnant animals (infection index 4.5-9.6, Brucella colonization $>200$ times than control) (14). However, the antigen-specific humoral and especially T-cell responses, which play an important role in the antibrucellosis immunity $(16,17)$, were comparable in both those studies. The Flu-BA_Omp19-SOD vaccine-induced antigen-specific IgG antibodies (IgG2a vs. IgG1), lymphocyte SI, and IFN- $\gamma$ production in sheep and goats at the first month PLV were less (SI: $2.3-2.7$ vs. 3.1-3.7; IFN- $\gamma$ production: $15.3-16.8$ vs. $19.1-19.4 \mathrm{ng} / \mathrm{mL}$ ) than those in published similar pregnant small ruminant study (14). We partly attribute the lower protective efficacy observed in this study to a wide range in age differences (6-18 months) in experimental animals used and small numbers included in each group $(n=5)$ compared to the findings in an earlier study (14). This assumption is consistent with previous work (18), wherein responses by PBMCs measured by two different assays between different sheep and within sheep over different sample time points varied substantially in terms of cytokine production and proliferation. In all previous experiments, animals used were more age-homogeneous, as well as in relatively larger numbers of younger animals (34 months, $n=7 /$ group or $9-10$ months, $n=9$ /group) $(12,14)$. Unfortunately, it has not been possible to reliably determine the effectiveness of the Flu-BA_Omp19-SOD vaccine on the adult immunized sheep and goats in this study. In any case, in this study, we used a sufficient number of animals in each group, which allowed us to obtain statistically reliable data.

At the third and sixth months PLV, we observed not only reduced Flu-BA_Omp19-SOD vaccine efficacy but also decreased humoral and T-cell responses. However, it is important to note that the severity of $B$. melitensis $16 \mathrm{M}$ infection in vaccinated sheep and goats at the indicated time of observation, estimated by the infection index and the degree of Brucella colonization from tissues, was significantly lower than in the control group. 
Mailybayeva et al.

Duration of Protection by a Brucellosis Vaccine

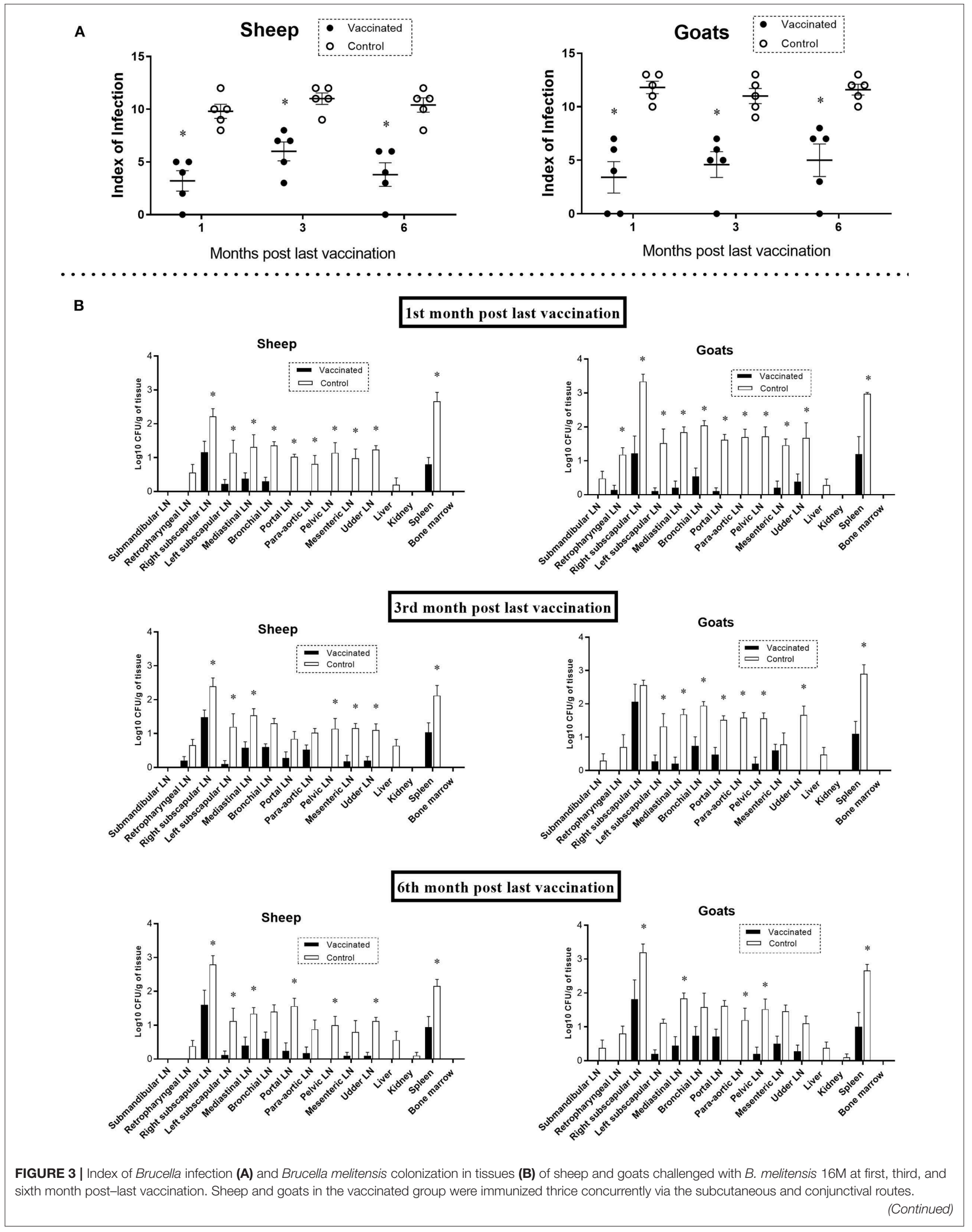

Frontiers in Veterinary Science | www.frontiersin.org

7

February 2020 | Volume 7 | Article 58 
FIGURE 3 | Animals in the control group were administered with adjuvant in phosphate-buffered saline. Animals were challenged with the virulent strain of $B$. melitensis $16 \mathrm{M}$ at a dose of $10^{6} \mathrm{CFU} /$ animal via subcutaneous route. The bacteriological examination was assessed by the index of infection in animals (number of organs and lymph nodes from which Brucella was isolated in each animal; the arithmetic mean \pm standard error was given) and colonization of Brucella in tissues (the data were given as $\log _{10} \mathrm{CFU} / \mathrm{g}$ ). Statistical analysis was performed using two-way analysis of variance followed by Sidak's multiple-comparisons test. The data of index of infection and colonization of $B$. melitensis in tissues are presented as mean \pm standard error; ${ }^{*} P=0.04-P<0.0001$ vaccine vs. control group. $L N$, lymph node.

Our results demonstrated that a partial protection was induced by the Flu-BA_Omp19-SOD vaccine in sheep and goats for at least 6 months PLV.

In comparison of our results on the duration of protective responses of the Flu-BA_Omp19-SOD vaccine in sheep and goats with the available vaccines $(19,20)$, it is clear that our candidate vaccine provided that it is insufficient to provide complete protection against infection. For example, the commercial vaccine (B. melitensis Rev.1) provides more than $80 \%$ full protection in vaccinated small ruminants for $\sim 2-5$ years (19). The Flu-BA vaccine after prime-boost immunization provides at least 12 months' antigen-specific T-cell immune response and protection in $57 \%$ of cattle against $B$. abortus 544 infection (20). The apparent difference in the duration of the protective antibrucellosis immune response in cattle (20) and small ruminants vaccinated with the same vaccine type indicates that IVV-based technology is most appropriate for cattle, and less so for sheep and goats. This can be explained by the fact that cattle are more sensitive to influenza A viruses $(7,8)$, and consequently, our IVVs effectively express Brucella proteins and induce a more pronounced immunity. Comparative analysis of all these data with the Flu-BA_Omp19-SOD vaccine $(12,14)$ indicates that it does not meet the important requirement of prolonged complete protective immunity in sheep and goats, indicating the need of improvements in the vaccine formulation, dosage, and immunization regimen. Further, it is important to note that the present study was performed using non-pregnant small ruminants, which are less sensitive to brucellosis infection (attributed to the presence of erythritol in the pregnant ruminant's placenta, an important growth factor of Brucella) $(21,22)$, and therefore expected higher vaccine efficacy than in pregnant animals, but the result was opposite. However, we still need to perform duration of protective immune responses to the Flu-BA_Omp19-SOD vaccine in pregnant sheep and goats. Consistent with our vaccine results, a similar attempt to use the commercial $B$. abortus vaccine RB51 in small ruminants against B. melitensis infection was unsuccessful (23).

To conclude, the Flu-BA_Omp19-SOD vaccine using improved formulation and administration method in sheep and goats provides augmented antigen-specific humoral and T-cell

\section{REFERENCES}

1. O'Callaghan D, Whatmore AM. Brucella genomics as we enter the multigenome era. Brief Funct Genomics. (2011) 10:334-41. doi: 10.1093/bfgp/elr026

2. Zinsstag J, Schelling E, Roth F, Bonfoh B, Savigny D, Tanner M. Human benefits of animal interventions for zoonosis control. Emerg Infect Dis. (2007) 13:527-31. doi: 10.3201/eid1304.060381 immune response lasting for only 1 month PLV and partial protection for 6 months against $B$. melitensis $16 \mathrm{M}$ infection.

\section{DATA AVAILABILITY STATEMENT}

All datasets generated for this study are included in the article/supplementary material.

\section{ETHICS STATEMENT}

This study was carried out in compliance with national and international laws and guidelines on animal handling. The protocol was approved by the Committee on the Ethics of Animal Experiments of the Research Institute for Biological Safety Problems of the Science Committee of the Ministry of Education and Science of the Republic of Kazakhstan. Animals were euthanized using sodium pentobarbital anesthetic and all recommended efforts were taken to minimize suffering.

\section{AUTHOR CONTRIBUTIONS}

KT, AM, SR, and NZ: conception and design of the study, or acquisition of data, or analysis and interpretation of data. KT: drafting the article or revising it critically for important intellectual content. KT, GR, and E-MZ: final approval of the version to be submitted.

\section{FUNDING}

This work was supported by grant from the Science Committee of the Ministry of Education and Science of the Republic of Kazakhstan (grant no. 1296/GF4 and AP05135949). The funders had no role in study design, data collection and analysis, decision to publish, or preparation of the manuscript.

\section{ACKNOWLEDGMENTS}

We thank B. Yespembetov, Zh. Kydyrbayev, Y. Kozhamkulov, D. Inkarbekov, M. Sarmykova, and N. Assanzhanova, employees of the Research Institute for Biological Safety Problems, for their assistance in the present study.
3. Garin-Bastuji B, Blasco JM, Grayon M, Verger JM. Brucella melitensis infection in sheep: present and future. Vet Res. (1998) 29:255-74.

4. Schurig GG, Sriranganathan N, Corbel MJ. Brucellosis vaccines: past, present and future. Vet Microbiol. (2002) 90:47996. doi: 10.1016/S0378-1135(02)00255-9

5. Moriyón I, Grilló MJ, Monreal D, González D, Marín C, López-Goñi I, et al. Rough vaccines in animal brucellosis: structural and genetic 
basis and present status. Vet Res. (2004) 35:1-38. doi: 10.1051/vetres: 2003037

6. Tabynov K. Influenza viral vector based Brucella abortus vaccine: a novel vaccine candidate for veterinary practice. Expert Rev Vaccines. (2016) 15:1237-9. doi: 10.1080/14760584.2016.1208089

7. Sreenivasan CC, Thomas M, Kaushik RS, Wang D, Li F. Influenza A in bovine species: a narrative literature review. Viruses. (2019) 11:E561. doi: 10.3390/v11060561

8. Reperant LA, Rimmelzwaan GF, Kuiken T. Avian influenza viruses in mammals. Rev Sci Tech Off Int Epiz. (2009) 28:13759. doi: 10.20506/rst.28.1.1876

9. Tibor A, Decelle B, Letesson JJ. Outer membrane proteins Omp10, Omp16, and Omp19 of Brucella spp are lipoproteins. Infect Immun. (1999) 67:49602. doi: 10.1128/IAI.67.9.4960-4962.1999

10. Oliveira SC, Zhu Y, Splitter GA. Sequence of the rpIJL operon containing L10 and L7/L12 genes from Brucella abortus. Gene. (1994) 140:1378. doi: 10.1016/0378-1119(94)90744-7

11. Bricker BJ, Tabatabai LB, Judge BA, Deyoe BL, Mayfield JE. Cloning, expression, and occurrence of the Brucella $\mathrm{Cu}-\mathrm{Zn}$ superoxide dismutase. Infect Immun. (1990) 58:2935-39. doi: 10.1128/IAI.58.9.2935-2939.1990

12. Tabynov K, Yespembetov B, Matikhan N, Ryskeldinova S, Zinina N, Kydyrbayev Z, et al. First evaluation of an influenza viral vector based Brucella abortus vaccine in sheep and goats: assessment of safety, immunogenicity and protective efficacy against Brucella melitensis infection. Vet Microbiol. (2016) 197:15-20. doi: 10.1016/j.vetmic.2016.11.001

13. Tabynov K, Sansyzbay A, Kydyrbayev Z, Yespembetov B, Ryskeldinova S, Zinina $\mathrm{N}$, et al. Influenza viral vectors expressing the Brucella OMP16 or L7/L12 proteins as vaccines against B. abortus infection. Virol J. (2014) 11:69. doi: 10.1186/1743-422X-11-69

14. Mailybayeva A, Yespembetov B, Ryskeldinova S, Zinina N, Sansyzbay A, Renukaradhya GJ, et al. Improved influenza viral vector based Brucella abortus vaccine induces robust $\mathrm{B}$ and $\mathrm{T}$-cell responses and protection against Brucella melitensis infection in pregnant sheep and goats. PLoS ONE. (2017) 12:e0186484. doi: 10.1371/journal.pone.0186484

15. Durán-Ferrer $M$, Léon L, Nielsen $K$, Caporale V, Mendoza J, Osuna A, et al. Antibody response and antigen-specific gammainterferon profiles of vaccinated and unvaccinated pregnant sheep experimentally infected with Brucella melitensis. Vet Microbiol. (2004) 100:219-31. doi: 10.1016/j.vetmic.2004.02.008

16. Jiang X, Baldwin CL. Effects of cytokines on intracellular growth of Brucella abortus. Infect Immun. (1993) 61:124-34. doi: 10.1128/IAI.61.1.124-134.1993
17. Oliveira SC, Splitter GA. CD8+ type 1 CD44hi CD45 RBloT lymphocytes control intracellular Brucella abortus infection as demonstrated in major histocompatibility complex class I- and class II-deficient mice. Eur J Immunol. (1995) 25:2551-7. doi: 10.1002/eji.1830250922

18. Wattegedera S, Sills K, Howard CJ, Hope JC, McInnes CJ, Entrican G. Variability in cytokine production and cell proliferation by mitogenactivated ovine peripheral blood mononuclear cells: modulation by interleukin (IL)-10 and IL-12. Vet Immunol Immunopathol. (2004) 102:6776. doi: $10.1016 /$ j.vetimm.2004.06.006

19. Díaz-Aparicio E1, Hernández L, Suárez-Güemes F. Protection against brucellosis in goats, five years after vaccination with reduced-dose Brucella melitensis Rev 1 vaccine. Trop Anim Health Prod. (2004) 36:11721. doi: 10.1023/B:TROP.0000012106.84833.3b

20. Tabynov K, Yespembetov B, Ryskeldinova S, Zinina N, Kydyrbayev Z, Kozhamkulov Y, et al. Prime-booster vaccination of cattle with an influenza viral vector Brucella abortus vaccine induces a long-term protective immune response against Brucella abortus infection. Vaccine. (2016) 34:43844. doi: 10.1016/j.vaccine.2015.12.028

21. Meador VP, Deyoe BL. Intracellular localization of Brucella abortus in bovine placenta. Vet Pathol. (1989) 26:513-5. doi: 10.1177/0300985889026 00609

22. Smith H, Williams AE, Pearce JH, Keppie J, Harris-Smith PW, FitzGeorge RB, et al. Foetal erythritol: a cause of the localization of Brucella abortus in bovine contagious abortion. Nature. (1962) 193:47-9. doi: 10.1038/193047a0

23. El Idrissi AH, Benkirane A, El Maadoudi M, Bouslikhane M, Berrada J, Zerouali A. Comparison of the efficacy of Brucella abortus strain RB51and Brucella melitensis Rev. 1 live vaccines against experimental infection with Brucella melitensis in pregnant ewes. Rev Sci Tech Off Int Epiz. (2001) 20:7417. doi: $10.20506 /$ rst.20.3.1305

Conflict of Interest: The authors declare that the research was conducted in the absence of any commercial or financial relationships that could be construed as a potential conflict of interest.

Copyright ( 2020 Mailybayeva, Ryskeldinova, Zinina, Zhou, Renukaradhya and Tabynov. This is an open-access article distributed under the terms of the Creative Commons Attribution License (CC BY). The use, distribution or reproduction in other forums is permitted, provided the original author(s) and the copyright owner(s) are credited and that the original publication in this journal is cited, in accordance with accepted academic practice. No use, distribution or reproduction is permitted which does not comply with these terms. 